\title{
Proceedings of the Conference 'Update in Spinal Medicine. Spasticity and the Neurogenic Bladder'
}

Held at Hungerford Hill Conference Centre, Pokolbin, New South Wales, Australia on 23-24 March 1984

The conference was organised by the Spinal Injury Unit of the Prince Henry Hospital, New South Wales and sponsored by the Pharmaceutical Division of CIBA-GEIGY Australia Ltd.

\section{Surgical approach to spasticity \\ Dr Ellis Griffiths \\ Spinal Injury Unit, Royal Perth Rehabilitation Hospital, Shenton Park, $W$. Australia 6008}

The surgical management of spasticity in three groups of patients, spinal cord injury, neurological impairment and neurosurgical trauma, is described. Emphasis is directed towards the means of reducing or ablating spasticity which can be of such severity as to disturb remaining function.

Methods employed are as follows:

1. Medication: the pharmaceuticals of choice are diazepam, baclofen, and dantrolene, each with its benefits and each with its drawbacks.

2. Physical therapy methods and cooling, which should be given a thorough trial over a prolonged period before embarking on radical measures.

3. Surgical relief of spasticity by direct approach to muscles and tendons, to nerves and to the central nervous system.

Methods of relieving spasm by surgical approach are described, especially tenotomy, tendon lengthening, tendon transfer procedures, release of contracted and spastic areas in the hip and in the knee, and neurectomies in the pelvis for gross adduction spasm. Release in the spastic upper limb is also described with a warning about an over-optimistic approach to surgery in these areas.

Surgery should be considered for relief of spasm only when all conservative measures, including medication, physical therapy and orthotic devices, have been tried and found inadequate to allow function, or to relieve spasm which will lead to gross contracture.

The gamma motoneurone: an over-rate entity in spinal injury Associate Professor David Burke Department of Neurology, Prince Henry Hospital, Little Bay, NSW 2036.

In spinal shock, there is hypoactivity of spinal cord reflex function. Muscle is 
said to lack tone, to be flaccid, and tendon jerks and cutaneous withdrawal reflexes are hyporesponsive.

It is generally considered that a critical factor in spinal shock is underactivity of the gamma efferent (fusimotor) system. As a result muscle spindle endings are believed to be deprived of a background drive that makes it possible for them to respond to tendon percussion or muscle stretch with sufficient intensity to produce a reflex contraction. Clearly, this belief requires that muscle spindle endings normally receive background fusimotor drive when the muscle is not contracting and that without the fusimotor drive muscle spindle endings are insensitive.

Direct recordings of muscle spindle afferent activity indicate that relaxed muscles receive no significant background fusimotor drive, and that muscle spindle endings are sufficiently responsive in the absence of such activity to produce a tendon jerk. In relaxed subjects muscle tone and tendon jerks do not depend on background fusimotor activity and spinal shock is not due to its withdrawal.

With the emergence from spinal shock, patients may develop the hyperactive tendon jerks and increased muscle tone of spasticity. Similar recordings of muscle spindle afferent activity have failed to demonstrate the increase in spindle reactivity that would suggest gamma motoneurone overactivity. Muscle tone and tendon jerks appear to be exaggerated because of a change in central reflex transmission rather than a peripheral mechanism operating on the muscle spindle.

\section{Lioresal: a fifteen-year report}

Associate Professor Richard F. Jones

Department of Rehabilitation Medicine, Prince Henry Hospital, Little Bay, NSW 2036

Prior to the introduction of Lioresal (baclofen), severe flexor spasms and spasticity were major problems for paraplegic and tetraplegic patients, reducing their ability to be independent in activities of daily living and in some cases causing them to be thrown from their chairs.

Trials of Lioresal at the Prince Henry Hospital since 1969 have demonstrated the safety and efficacy of the drug as a spasmolytic agent. The starting dosage is $40 \mathrm{mg}$ per day in divided doses, and the dose is gradually increased until an optimal effect is seen. Rapid increase in dose may result in cerebral side effects such as nightmares, confusional states and sedation. Lioresal is generally well tolerated and dosages of up to $150 \mathrm{mg}$ per day have been used without adverse effects, though generally a maximum of $100 \mathrm{mg}$ per day is recommended. Dosage can be titrated as a compromise between the spastic muscle splint and residual muscle function.

Action on the striated muscle of the external sphincter has been demonstrated and clinical trials have indicated that the drug has a place in the management of the neuropathic bladder.

In Australia approximately 2500 people are currently taking Lioresal. The advent of Lioresal represents the greatest addition to the therapeutic armamentarium against spasticity in the last 15 years. 
Lioresal: the west australian experience

Dr John Ker

Spinal Injury Unit, Royal Perth Rehabilitation Hospital, Shenton Park, WA 6008

Baclofen (Lioresal) as an anti-spasmodic agent has been in use in the Spinal Cord Injuries Unit of Royal Perth (Rehabilitation) Hospital since 1976.

Published clinical studies (when comparing the clinical effects of Lioresal and diazepam for the control of spasticity in spinal cord patients), indicate little difference in the efficacy of either agent in reducing frequency of spasms, decreased resistance to passive movements and decreased force of clonic movements. Lioresal, however, does not produce the somnolence, muscle weakness, ataxia, vertigo and possible long term habituation associated with diazepam.

Early in the introduction of Lioresal in the Royal Perth (Rehabilitation) Hospital a group of four patients experienced significant undesirable complications in their treatment. The case histories of these patients who presented with blepharitis and acute organic psychosis, and two cases of epilepsy have been documented.

Diazepam has clear advantages for the treatment of bed-bound patients who require early and adequate control of pharmacological means of their spasticity. However, for the mobile patient, whether wheelchair bound or ambulant, Lioresal has significant advantages.

\section{Lioresal: too much of a good thing?}

Dr Hugh Dickson and Dr Stella Engel

The Prince Henry Hospital, Little Bay, NSW 2036

Baclofen (Lioresal) exerts a powerful inhibitory influence on spinal reflex activity. It is rapidly and completely absorbed after oral ingestion and excretion is primarily renal. In therapeutic doses spinal effects predominate though sedation and confusional states may appear, especially in the elderly or in those who dose has been too rapidly increased. Cerebral depression becomes increasingly marked as the dose increases.

Suicidal attempts with baclofen occur periodically. The constant features of baclofen overdosage are:

1. Loss of or depression of consciousness

2. Respiratory depression

3. Hypotonia

4. Hyporeflexia or areflexia.

Inconstant features include bradycardia, hypotension, pupillary changes, hypothermia, myoclonus, major seizures and retrograde amnesia. Transient tachycardia and hypertension have been reported as late as 3 days after overdosage.

From 1974-1984 three confirmed cases of intentional overdosage have been documented in patients of The Prince Henry Hospital Spinal Unit. Dosages ranged between $250 \mathrm{mg}$ and $1000 \mathrm{mg}$. All patients recovered rapidly with supportive treatment and there were no permanent sequelae. 


\section{Emotional concomitants of spasm in spinal cord injury patients Dr George Rawson \\ Spinal Injury Unit, Morris Hospital, Northfield, SA 5085}

Spasticity, the legacy of spinal injury is associated with spasm of the skeletal musculature. Psychological re-adjustments following spinal cord injury relate to many of its sequelae. The occurrence of spasms in the rehabilitation of spinal cord injury should raise questions about the patient's emotional adaptation, and also interest in the responses to this neurological symptom. An examination of the literature shows a dearth of detailed information on the relationship of spasm and spasticity to emotional factors in spinal cord rehabilitees.

This paper poses questions concerning the spasm-emotion interdependency, and suggests that psychological factors may be involved in the aggravation of spasm mediated by the complex interplay of personality socio-cultural, and psycho-physiological mechanisms involved in the rehabilitation process.

A spasm questionnaire survey of inpatient and outpatient spinal rehabilitees together with some clinical data suggests that spasm is not an insignificant problem in spinal cord patients, even years following injury; also that the anhedonic emotional responses anger, anxiety, frustration and depression are related to a subjective increase in frequency of spasm with disruption in mobility and activities of daily living.

The adoption of the Learning or Education Model in Rehabilitation, as proposed by Roberta Trieschmann, is presented as a prophylactic to the development of spinal injury complications. The effective treatment of spasm demands attention to the total person. Modern spasmolytic and other techniques have alleviated much of the suffering of the past decade but psychological factors should be given appropriate consideration in this area of spinal injury.

\section{Prevention of oedema and contracture and its relationship to spasticity}

Sir George Bedbrook

13 Colin Grove, West Perth, Western Australia 6005

Oedema and contractures in the feet of spinal paralytics are insidious complications increasing in incidence during long-term management.

A group of 50 patients was assessed, 42 of whom manifested spasm.

All these cases showed oedema associated with joint limitation including ankle, tarsal (inter and mid), subtalar and toes. This was noted particularly in those with upper motor neuron lesions.

Prevention means persistence with measures well known in acute care areas. 


\section{Recent advances in the management of spina bifida: to inflate or not to inflate}

Dr R. Farnsworth

Department of Urology, The Prince Henry Hospital, Little Bay, NSW 2036

Two major objectives in the management of the urinary tract in children with spina bifida are the preservation of renal function and the attainment of optimal urinary control. While derangements of bladder function in children with spina bifida differ significantly from those in spinal injured patients, both groups have detrusor sphincter dyssynergia as the major underlying cause of renal damage; in both groups identification of the precise mechanism of incontinence is necessary to achieve optimal urinary control.

Each child with spina bifida has a unique neurological deficit with varying degrees of preservation of ascending and descending tracts in the cord and varying degrees of disruption of the centre for micturition, reflex arcs and somatic innervation of the external sphincter mechanism. Pressure recording studies of bladder function have in the last 15 years been the single most important determining factor guiding our management in relation to protection of the upper urinary tract, by detecting detrusor sphincter dyssynergia and evaluating and managing urinary incontinence.

Methods of management of detrusor sphincter dyssynergia include overdilatation of the female urethra, sphincterotomy in the male and intermittent catheterisation. Pharmacological techniques have been less successful and urinary diversion is considered a last resort, accounting for five children only in our series of over 200 .

Incontinence control methods include intermittent catheterisation, drugs modifying bladder and urethral function, either separately or in combination with catheterisation, and Katona's method of bladder stimulation. Implantation of an artificial urinary sphincter is being increasingly used, case selection depending on urodynamic findings as the failure rate increases significantly in those children with persistent outlet obstruction and detrusor hyperreflexia. 